\title{
Tumour motion management in lung cancer: a narrative review
}

\author{
Angela Botticella ${ }^{1}$, Antonin Levy ${ }^{1,2,3}$, Guillaume Auzac ${ }^{1}$, Isabelle Chabert ${ }^{1}$, Céline Berthold ${ }^{1}$, \\ Cécile Le Pechoux ${ }^{1}$
}

${ }^{1}$ Department of Radiation Oncology, Institut d'Oncologie Thoracique (IOT), Gustave Roussy, F-94805, Villejuif, France; ${ }^{2}$ Univ Paris Sud, Université Paris-Saclay, F-94270, Le Kremlin-Bicêtre, France; ${ }^{3}$ INSERM U1030, Molecular Radiotherapy, Gustave Roussy, Université Paris-Saclay, F-94805, Villejuif, France

Contributions: (I) Conception and design: A Botticella; (II) Administrative support: None; (III) Provision of study materials or patients: A Levy, C Le Pechoux; (IV) Collection and assembly of data: None; (V) Data analysis and interpretation: None; (VI) Manuscript writing: All authors; (VII) Final approval of manuscript: All authors.

Correspondence to: Angela Botticella, MD. Department of Radiation Oncology, Gustave Roussy; 114, Rue Edouard Vaillant, Villejuif, France.

Email: angela.botticella@gustaveroussy.fr.

\begin{abstract}
Respiratory motion is one of the geometrical uncertainties that may affect the accuracy of thoracic radiotherapy in the treatment of lung cancer. Accounting for tumour motion may allow reducing treatment volumes, irradiated healthy tissue and possibly toxicity, and finally enabling dose escalation. Historically, large population-based margins were used to encompass tumour motion. A paradigmatic change happened in the last decades led to the development of modern imaging techniques during the simulation and the delivery, such as the 4-dimensional (4D) computed tomography (CT) or the 4D-cone beam CT scan, has contributed to a better understanding of lung tumour motion and to the widespread use of individualised margins (with either an internal tumour volume approach or a mid-position/ventilation approach). Moreover, recent technological advances in the delivery of radiotherapy treatments (with a variety of commercial solution allowing tumour tracking, gating or treatments in deep-inspiration breathhold) conjugate the necessity of minimising treatment volumes while maximizing the patient comfort with less invasive techniques. In this narrative review, we provided an introduction on the intra-fraction tumour motion (in both lung tumours and mediastinal lymph-nodes), and summarized the principal motion management strategies (in both the imaging and the treatment delivery) in thoracic radiotherapy for lung cancer, with an eye on the clinical outcomes.
\end{abstract}

Keywords: Lung cancer; motion management; 4-dimensional computed tomography (4D-CT); deep-inspiration breath-hold (DIBH); gating

Submitted Jul 15, 2020. Accepted for publication Apr 23, 2021.

doi: $10.21037 /$ tlcr-20-856

View this article at: http://dx.doi.org/10.21037/tlcr-20-856

\section{Introduction}

A variety of geometrical uncertainties may affect the accuracy of lung cancer radiation therapy (RT), thus influencing the accuracy of imaging, treatment planning and treatment delivery: respiratory motion, tumour delineation, microscopic disease definition, inter-fraction setup errors and baseline shifts (1). Those inaccuracies are in generally overcome by applying safety margins around the target, in order to generate the planning target volume (PTV) (1).
However, larger margins result in increased irradiated healthy tissue, possibly leading to increased toxicity.

Motion-management is of pivotal importance in reducing geometrical uncertainties in lung RT. The European Organisation for the Research and the Treatment of Cancer (EORTC) guidelines recommend the adoption of motion management strategies, in order to avoid the sue of free breathing computed tomography (CT) scans in the treatment planning process, because it may capture the random position of a tumour and/or artefacts (2). 
According to the American Association of Physicists in Medicine (AAPM) (3), if the magnitude of the motion is very small $(<5 \mathrm{~mm})$, the extra effort of using a respiratory management technique is "unwarranted, unless significant normal tissue sparing can be gained with the respiratorymanagement technique". The 5 -mm threshold was used because it can generate significant motion artefacts or systematic errors (3), and can be reduced when stereotactic body radiotherapy (SBRT) is performed.

The main aim of this narrative review is to describe intra-fraction tumour motion and summarize the principal motion management strategies in thoracic radiotherapy for lung cancer.

We present the following article in accordance with the narrative review reporting checklist (available at http:// dx.doi.org/10.21037/tlcr-20-856).

\section{Methods}

A formal literature search was not carried out. Published studies were identifies through "pearl growing" and citation chasing. Only studies in English published after 2000 were included.

\section{Measuring tumour respiratory motion}

Respiratory motion is a complex mechanism, affected by various patient-related (i.e., interstitial fibrosis, lung emphysema, pleural effusion, previous thoracic surgery/ RT or chemotherapy, muscular fatigue) and tumourrelated factors (i.e., tumour location, for example: upper lobes versus lower lobes, proximity to the heart) (4). The extent of respiratory movements in lung cancer has been detailed by Seppenwoolde et al. (5): the authors inserted gold markers in 20 patients into or near the tumour mass, and tracked their motion using a fluoroscopic real-time tumour tracking system able to determine the $3 \mathrm{D}$ position of the tumour by performing 30 images per second. The conclusions were that the average amplitude of tumour motion was the greatest in the cranial-caudal direction for tumours located in the lower lobe and not attached to rigid structures (such as chest wall and vertebrae) compared to upper lobe and attached to rigid structures $[12 \pm 6 \mathrm{~mm}(\mathrm{SD})$ versus $2 \pm 2 \mathrm{~mm}(\mathrm{SD})]$. The lateral and anterior-posterior movement was small in all cases $[1.2 \pm 0.9 \mathrm{~mm}(\mathrm{SD})$ for the left-right direction and $2.2 \pm 1.9 \mathrm{~mm}(\mathrm{SD})$ for the anteriorposterior direction]. The time-averaged position was closer to the exhale position, because the tumour spent more time in the exhalation phase than in the inhalation phase. In the average trajectory of the tumour, hysteresis was observed in $50 \%$ of the patients: the tumour followed a different path during the inhalation than during the exhalation. The breathing technique (diaphragm and chest breathing combined asymmetrically), lung's dynamic properties (the tumour motion may be delayed compared to the motion of the chest wall and the diaphragm) and even the heartbeat may influence a measurable tumour motion. Finally, in the case of lung cancer with mediastinal node involvement, it has been showed that the movement of the primary tumours and the lymph nodes is not necessarily in the same phase (6). Pantarotto et al. (6) examined the pattern of movement of 100 lymph nodes from 41 patients and concluded that the magnitude of the nodal motion was often $>5 \mathrm{~mm}$, mostly in cranial-caudal direction and strongly associated with nodal station (with lower mediastinal nodes showing the highest motion). Phase offsets between the primary tumour and the lymph nodes were minimal at end-inspiration (phase offset of one phase in $12 \%$ of nodes) and maximal at endexpiration (phase offset of one phase of $33 \%$ and $12 \%$ of two phases).

In a study on intra-fractional variability of tumour position and tumour motion (27 lesions and 66 treatment fractions), Guckenberger et al. (7) showed that intra-fraction tumour drift was $<48 \mathrm{~mm}$ in $90 \%$ of all fractions, with a maximum tumour drift of $72 \mathrm{~mm}$. No correlation was found between any clinical, planning or treatment parameter and intra-fractional tumour position variability.

\section{How to deal with respiratory motion?}

\section{Respiratory correlated imaging, individualized and population margins}

\section{Respiratory correlated imaging}

In order to reduce the artefacts induced by respiratory motion, several groups developed respiratory-correlated acquisition techniques (8). A 4-dimensional (4D) CT dataset is obtained from 3D images at multiple phases of the respiratory cycle by retrospectively sorting the data using the information from the patient's breathing cycle. The 4D-CT is to date considered the standard to gain detailed information about range and pattern of breathing respiratory motion (2).

Motion compensated cone beam CT (MC-CBCT) have been developed as well (9).

However, a 4D-CT or an MC-CBCT still contain 
artefacts due to residual breathing irregularities and are only a "snapshot" of the patient's breathing behaviour.

Steiner et al. (10) showed an under-prediction of the target motion in 4D-CT end 4D CBCT in all directions: the errors were larger in the inhale phases and smaller in the end-exhale phase. The authors showed that a $5-\mathrm{mm}$ margin was sufficient to cover the 4D-CT-based motion measurements in $76 \%$ of the treatment fractions, with short times of exceeding motion (ranging from $1-20 \%$ of the treatment time).

Wolthaus et al. (11) developed a mid-ventilation approach to exploit the 4D-CT data, by extracting a single frame of $4 \mathrm{D}-\mathrm{CT}$ where the tumour is the closest to its time-averaged position (the mid-ventilation CT). In order to limit the breathing artefacts derived by using a singleframe image, the same authors (in a subsequent publication) developed a mid-position approach, by deforming all the phases of the $4 \mathrm{D}-\mathrm{CT}$ in order to produce a single image representing the time-weighted position of all images (12). Respiratory motion, similarly to random errors, blur the cumulative dose distribution, with limited effect on final dose distribution (13).

\section{Individualized margins}

Separate margins have been proposed for tumour motion (internal margins) and setup (setup margins). The use of an internal target volume (ITV) encompasses motion and shape changes over the respiratory cycle, based on a composite delineation on an inspiration and expiration breath-hold scans, all/several phases of a 4D-CT or on a $4 \mathrm{D}-\mathrm{CT}$ maximal intensity projection (1). The ITV is expanded to the PTV to account for setup margins. Even with an accurate set-up, internal displacement of the tumour may be significant: Hansen et al. (14) reported a standard deviation of the overall tumour displacement of respectively 2,3 and $4 \mathrm{~mm}$ in the left-right, anterior-posterior and cranial-caudal directions. Set-up based on volumetric CBCT has now become the standard of care (15). The use of daily CBCT could minimize the ITV-PTV setup margins. Grills et al. (16) showed that the margins required to account for setup error alone would be $9 \mathrm{~mm}$ (right-left and anterior-posterior) and $13 \mathrm{~mm}$ (cranial-caudal) when using a stereotactic body frame (without image guidance). However, when image guidance (CBCT) was added to the body frame, the setup margins were reduced to $1-2 \mathrm{~mm}$ (residual error alone) or 2-4 $\mathrm{mm}$ (residual error + intrafraction drift).

The recently developed 4D-CBCT (9) and the gated
CBCT (17) may reduce the motion artefacts and image blurring.

Limitations of the 4D-CT include motion artefacts, irregular breathing patterns and the rigorous quality assurance (18).

\section{Population margins}

Geometrical uncertainties can be incorporated into the treatment planning process by taking population-based margins around the clinical target volume (CTV), in order to define the PTV. Amongst others, the so-called "van Herk recipe" calculates the margin around the CTV that is needed to deliver at least $95 \%$ of the prescribed dose to $90 \%$ of the patients (13). To calculate the CTV to PTV margin, apart from the SD (standard deviation) of the systematic and random errors, the width of the penumbra modelled by a cumulative Gaussian and the inverse cumulative standard-normal distribution at the prescribed PTV minimum dose level. In the lung where in photon therapy the increased range of secondary electrons results in a broadening of the beam penumbra, the additional margin for random errors is small. Systematic errors therefore have a dominant effect on the cumulative dose, especially in the lungs.

\section{Management of the intra-fraction movement: breath-bold techniques, gating and tracking}

When toxicity is of particular concern, the reduction of the respiratory motion can be achieved by irradiating the target when only in a pre-defined phase (breath-hold techniques) or window (gating) of its breathing trajectory or by tracking the tumour throughout the treatment in free breathing (tracking) (19).

\section{Breath-hold techniques}

In the deep-inspiration breath-hold (DIBH), the patient attempted a maximum inhalation during simulation and treatment, by using spirometer linked to a video glasses or a screen (20). This technique allows the displacement of heart and lung from the high-dose region $(21,22)$. Rosenzweig et al. (23) showed that normal tissue complication in lung decreased with the DIBH technique compared with freebreathing. However, a limitation of this technique is patient compliance: Mah et al. (24) showed that only 50\% of the lung cancer patient in their study could perform this method.

In order to increase the stability of the breath-hold 
procedures, the Active Breathing Coordinator ${ }^{\mathrm{TM}}$ (ABC, Elekta, Stockholm Sweden) was developed: this system uses a combination of a valve system shutting off airflow and a visual guidance to the patient. Breath-hold during expiration is more stable (and more comfortable for the patient) than during inspiration, but the choice of using expiration or inspiration breath-hold will depend from dosimetric concerns. A compromise could be to use a moderate DIBH. Panakis et al. (25) showed that the use of the $\mathrm{ABC}$ system was associated with a mean reduction in physical lung parameters of $18-25 \%$ compared to the freebreathing.

The main drawback of these techniques is the patient compliance and reproducibility, which can be enhanced by patient training and coaching, using audio and video guidance (26-29). Inter-fraction variations can be significant (mostly in the cranial-caudal direction; mean variation in the GTV centre of mass: $5.1 \mathrm{~mm}$ ), and could be limited by using daily image-guided RT (IGRT) (30), such as daily CBCT, with soft-tissue registration (31).

\section{Gating}

In the respiratory gating, the beam is turned on and off in synchronization the breathing cycle, and the target is irradiated only when in a pre-defined window (the so called "gating window") of the breathing cycle. A breathing monitoring device for providing the trigger signal is required, and both internal fiducial markers or external surrogates (external optical skin markers, pressure sensors or abdominal belts) can be used (32). The correspondence between the surrogate motion and the target motion should be established before the treatment and verified regularly, in order to avoid the underdosage of the tumour $(3,33)$. The comparison between external and internal surrogates shows a superiority of the latter (34).

The gating can be phase-based (delivering is planned in a specific phase) or amplitude-based (delivery is in a window, where the maximum and minimum limits are pre-defined).

\section{Tracking}

Recent technological advances in radiotherapy delivery allowed developing solutions that can beam continuously at the moving target.

As said before, the gating and the tracking are based on the assumption that the tumour motion can be predicted using surrogates (internal or external). In addition, tracking relies on a prediction model of the respiratory motion, which can anticipate the future position of the tumour.
Real-time motion compensation was firstly implemented in 2002 with the Cyberknife System ${ }^{\mathrm{TM}}$ (Accuray, Sunnyvale, CA, USA). The robotic arm of the Cyberknife is programmed to move synchronously with the breathing cycle, in a trajectory following the projected $3 \mathrm{D}$ motion of the target. The target motion is not monitored directly: a series of orthogonal radiographic images is acquired (by two orthogonal cameras) before the starting of the treatment and a mathematical model between the target motion and the motion of a set of surface markers is generated. During the beam on phase, intermittent orthogonal images are acquired, in order to verify the target position and eventually correct the model.

Four image guidance protocols are used for lung tumours treated with the Cyberknife System ${ }^{\mathrm{TM}}$ : (I) synchrony tracking of fiducial markers (placed in the tumour or close to the tumour); (II) XSight lung tracking (direct tracking of the tumour by two cameras or one camera, which is fiducial-less); (III) Xsight spine tracking (tracking of the adjacent vertebral body) (35). The use of the fiducials is associated with a risk of pneumothorax ranging between $18 \%$ and $25 \%(36,37)$, with fiducial displacement/migration and longer treatment delays (38).

The XSight spine does not really track the movement of the tumour during treatment, and it requires a larger ITV (accounting for the tumour motion in the different phases of the respiratory cycle). Fiducial-less treatments performed with Xsight lung may allow tumour tracking (by the means of soft-tissue tracking). This tracking modality needs adequate tumour visualisation, which must be assessed with a tumour visualisation test performed before the treatment. In case of negative test (tumour not well visualised), the patient has to be addressed to another treatment system. In a retrospective series, Bahig et al. (38) showed that XSight lung tumour visualisation test was satisfactory in $66 \%$ of patients, with larger tumour volume and higher tumour density being predictive factors of a successful test.

No randomised prospective data comparing the clinical outcome of different tracking techniques has been published (and such trials are virtually impossible: few institutions have this choice in terms of equipment). However, Claude et al., in an observational prospective multicentric study, found no differences in terms of local control, progressionfree survival, overall survival and toxicity in two cohorts treated either with a Linac- or a Cyberknife-delivered SBRT (39).

Dynamic multileaf collimator (MLC) tracking for a standard gantry-based Linac has been recently used for 
prostate cancer, with potential applications also in lung cancer treatment (40).

The recent development of an magnetic resonance imaging (MRI)-Linac hybrid technology allow to combine real-time tracking and MR-guided daily adaptive workflow, and is currently under investigation in few selected centres (41).

\section{Main remaining challenges}

Very few studies reporting the clinical benefits of respiratory management have been published (and none of them is randomized). The issue of the choice of the optimal system is rather complex. In the decision-making process, several factors need to be considered, the most significant being the available technical equipment of the radiotherapy department and the patient selection (with an individualized estimate of the benefit of tumour management strategy on each patient on the basis of the amplitude of the breathing motion or the regularity of the breathing). The regularity of the breathing determines the success of respiratory management: the more regular the pattern, the more reliable is model built on the target motion and the external surrogates that guide the beam position.

Many challenges should be solved in the future, with the increased use of proton therapy in thoracic oncology (42), dose-painting and adaptive RT. In this review, the motion management in combination with protons has not been elaborated, but it has been the subject of a number of papers [see $(43,44)$ for the evaluation of plan robustness evaluation and the potential interplay effect].

Further analyses are required to confirm the impact of tumour management on the therapeutic index (45), especially for patients' systemic treatments [i.e., cardiotoxic chemotherapy (46)].

\section{Summary}

Tumour motion management remains a pivotal challenge for lung cancer treatment

Due to a very limited literature comparing the outcomes of patients treated with different motion management strategies, it is quite difficult to quantify the real impact of novel technologies on the therapeutic window.

Clinical trials investigating the best strategy for a given clinical presentation and for a given patient are needed. More widely available technical solutions are awaited.

\section{Acknowledgments}

Funding: None.

\section{Footnote}

Provenance and Peer Review: This article was commissioned by the guest editors (Jacek Jassem and Rafal Dziadziuszko) for the series "Radiotherapy in thoracic malignancies" published in Translational Lung Cancer Research. The article has undergone external peer review.

Reporting Checklist: The authors have completed the Narrative Review reporting checklist. Available at http:// dx.doi.org/10.21037/tlcr-20-856

Peer Review File: Available at http://dx.doi.org/10.21037/ tlcr-20-856

Conflicts of Interest: All authors have completed the ICMJE uniform disclosure form (available at http://dx.doi. org/10.21037/tlcr-20-856). The series "Radiotherapy in thoracic malignancies" was commissioned by the editorial office without any funding or sponsorship. AL serves as an unpaid editorial board member of Translational Lung Cancer Research from Sep 2019 to Sep 2021. The authors have no other conflicts of interest to declare.

Ethical Statement: The authors are accountable for all aspects of the work in ensuring that questions related to the accuracy or integrity of any part of the work are appropriately investigated and resolved.

Open Access Statement: This is an Open Access article distributed in accordance with the Creative Commons Attribution-NonCommercial-NoDerivs 4.0 International License (CC BY-NC-ND 4.0), which permits the noncommercial replication and distribution of the article with the strict proviso that no changes or edits are made and the original work is properly cited (including links to both the formal publication through the relevant DOI and the license). See: https://creativecommons.org/licenses/by-nc-nd/4.0/.

\section{References}

1. Sonke JJ, Belderbos J. Adaptive radiotherapy for lung cancer. Semin Radiat Oncol 2010;20:94-106.

2. De Ruysscher D, Faivre-Finn C, Nestle U, et al. European 
Organisation for Research and Treatment of Cancer recommendations for planning and delivery of high-dose, high-precision radiotherapy for lung cancer. J Clin Oncol 2010;28:5301-10

3. Keall PJ, Mageras GS, Balter JM, et al. The management of respiratory motion in radiation oncology report of AAPM Task Group 76. Med Phys 2006;33:3874-900.

4. Shirato H, Seppenwoolde Y, Kitamura K, et al. Intrafractional tumor motion: lung and liver. Semin Radiat Oncol 2004;14:10-8.

5. Seppenwoolde Y, Shirato H, Kitamura K, et al. Precise and real-time measurement of $3 \mathrm{D}$ tumor motion in lung due to breathing and heartbeat, measured during radiotherapy. Int J Radiat Oncol Biol Phys 2002;53:822-34.

6. Pantarotto JR, Piet AHM, Vincent A, et al. Motion analysis of 100 mediastinal lymph nodes: potential pitfalls in treatment planning and adaptive strategies. Int J Radiat Oncol Biol Phys 2009;74:1092-9.

7. Guckenberger M, Meyer J, Wilbert J, et al. Intra-fractional uncertainties in cone-beam CT based image-guided radiotherapy (IGRT) of pulmonary tumors. Radiother Oncol 2007;83:57-64.

8. Lagerwaard FJ, Van Sornsen de Koste JR, Nijssen-Visser MRJ, et al. Multiple "slow" CT scans for incorporating lung tumor mobility in radiotherapy planning. Int J Radiat Oncol Biol Phys 2001;51:932-7.

9. Sonke JJ, Zijp L, Remeijer P, et al. Respiratory correlated cone beam CT. Med Phys 2005;32:1176-86.

10. Steiner E, Shieh C, Caillet V, et al. Both four-dimensional computed tomography and four-dimensional cone beam computed tomography under-predict lung target motion during radiotherapy. Radiother Oncol 2019;135:65-73.

11. Wolthaus JWH, Schneider C, Sonke JJ, et al. Midventilation CT scan construction from four-dimensional respiration-correlated CT scans for radiotherapy planning of lung cancer patients. Int J Radiat Oncol Biol Phys 2006;65:1560-71.

12. Wolthaus JWH, Sonke JJ, van Herk M, et al. Reconstruction of a time-averaged midposition CT scan for radiotherapy planning of lung cancer patients using deformable registration. Med Phys 2008;35:3998-4011.

13. van Herk M, Remeijer $P$, Rasch $C$, et al. The probability of correct target dosage: dose-population histograms for deriving treatment margins in radiotherapy. Int J Radiat Oncol Biol Phys 2000;47:1121-35.

14. Hansen AT, Petersen JB, Høyer M. Internal movement, set-up accuracy and margins for stereotactic body radiotherapy using a stereotactic body frame. Acta Oncol
2006;45:948-52.

15. Jaffray DA, Siewerdsen JH, Wong JW, et al. Flatpanel cone-beam computed tomography for imageguided radiation therapy. Int J Radiat Oncol Biol Phys 2002;53:1337-49.

16. Grills IS, Hugo G, Kestin LL, et al. Imageguided radiotherapy via daily online cone-beam CT substantially reduces margin requirements for stereotactic lung radiotherapy. Int J Radiat Oncol Biol Phys 2008;70:1045-56.

17. Kincaid RE Jr, Yorke ED, Goodman KA, et al. Investigation of gated cone-beam CT to reduce respiratory motion blurring. Med Phys 2013;40:041717.

18. Jiang SB, Wolfgang J, Mageras GS. Quality assurance challenges for motion-adaptive radiation therapy: gating, breath holding, and four-dimensional computed tomography. Int J Radiat Oncol Biol Phys 2008;71:S103-7.

19. Hugo GD, Campbell J, Zhang T, et al. Cumulative lung dose for several motion management strategies as a function of pretreatment patient parameters. Int J Radiat Oncol Biol Phys 2009; 74:593-601.

20. Boda-Heggemann J, Knopf AC, Simeonova-Chergou A, et al. Deep Inspiration Breath Hold - Based Radiation Therapy: A Clinical Review. Int J Radiat Oncol Biol Phys 2016;94:478-92.

21. Barnes EA, Murray BR, Robinson DM, et al. Dosimetric evaluation of lung tumor immobilization using breath hold at deep inspiration. Int J Radiat Oncol Biol Phys 2001;50:1091-8.

22. Persson GF, Scherman Rydhög J, Josipovic M, et al. Deep inspiration breath-hold volumetric modulated arc radiotherapy decreases dose to mediastinal structures in locally advanced lung cancer. Acta Oncol 2016;55:1053-6.

23. Rosenzweig KE, Hanley J, Mah D, et al. The deep inspiration breath-hold technique in the treatment of inoperable non-small-cell lung cancer. Int J Radiat Oncol Biol Phys 2000;48:81-7.

24. Mah D, Hanley J, Rosenzweig KE, et al. Technical aspects of the deep inspiration breath-hold technique in the treatment of thoracic cancer. Int J Radiat Oncol Biol Phys 2000;48:1175-85.

25. Panakis N, McNair HA, Christian JA, et al. Defining the margins in the radical radiotherapy of non-small cell lung cancer (NSCLC) with active breathing control (ABC) and the effect on physical lung parameters. Radiother Oncol 2008;87:65-73.

26. George R, Chung TD, Vedam SS, et al. Audio-visual biofeedback for respiratory-gated radiotherapy: impact 
of audio instruction and audio-visual biofeedback on respiratory-gated radiotherapy. Int J Radiat Oncol Biol Phys 2006;65:924-33.

27. Damkjær SMS, Aznar MC, Pedersen AN, et al. Reduced lung dose and improved inspiration level reproducibility in visually guided DIBH compared to audio coached EIG radiotherapy for breast cancer patients. Acta Oncol 2013;52:1458-63.

28. Josipovic M, Aznar MC, Thomsen JB, et al. Deep inspiration breath hold in locally advanced lung cancer radiotherapy: validation of intrafractional geometric uncertainties in the INHALE trial. Br J Radiol 2019;92:20190569.

29. Giraud P, Morvan E, Claude L, et al. Respiratory gating techniques for optimization of lung cancer radiotherapy. J Thorac Oncol 2011;6:2058-68.

30. Brock J, McNair HA, Panakis N, et al. The use of the active breathing coordinator throughout radical nonsmall-cell lung cancer (NSCLC) radiotherapy. Int J Radiat Oncol Biol Phys 2011;81:369-75.

31. Ottosson W, Rahma F, Sjöström D, et al. The advantage of deep-inspiration breath-hold and cone-beam CT based soft-tissue registration for locally advanced lung cancer radiotherapyDIBH radiotherapy for lung cancer patients. Radiother Oncol 2016;119:432-7.

32. Cole AJ, Hanna GG, Jain S, et al. Motion management for radical radiotherapy in non-small cell lung cancer. Clin Oncol (R Coll Radiol) 2014;26:67-80.

33. Korreman SS, Juhler-Nøttrup T, Boyer AL. Respiratory gated beam delivery cannot facilitate margin reduction, unless combined with respiratory correlated image guidance. Radiother Oncol 2008;86:61-8.

34. Korreman S, Mostafavi H, Le QT, et al. Comparison of respiratory surrogates for gated lung radiotherapy without internal fiducials. Acta Oncol 2006;45:935-42.

35. Loo BWJ, Kavanagh BD, Meyer JL. Motion management and image guidance for thoracic tumor radiotherapy: clinical treatment programs. Front Radiat Ther Oncol 2011;43:271-91.

36. Lischalk JW, Woo SM, Kataria S, et al. Long-term outcomes of stereotactic body radiation therapy (SBRT) with fiducial tracking for inoperable stage I non-small cell lung cancer (NSCLC). J Radiat Oncol 2016;5:379-87.

37. Collins BT, Vahdat S, Erickson K, et al. Radical cyberknife radiosurgery with tumor tracking: an effective treatment for inoperable small peripheral stage I non-small cell lung cancer. J Hematol Oncol 2009;2:1.

38. Bahig H, Campeau MP, Vu T, et al. Predictive parameters of CyberKnife fiducial-less (XSight Lung) applicability for treatment of early non-small cell lung cancer: a single-center experience. Int J Radiat Oncol Biol Phys 2013;87:583-9.

39. Claude L, Morelle M, Mahé MA, et al. A comparison of two modalities of stereotactic body radiation therapy for peripheral early-stage non-small cell lung cancer: results of a prospective French study. Br J Radiol 2020;93:20200256.

40. Keall PJ, Cattell H, Pokhrel D, et al. Geometric accuracy of a real-time target tracking system with dynamic multileaf collimator tracking system. Int J Radiat Oncol Biol Phys 2006;65:1579-84.

41. van Sörnsen de Koste JR, Palacios MA, Bruynzeel AME, et al. MR-guided Gated Stereotactic Radiation Therapy Delivery for Lung, Adrenal, and Pancreatic Tumors: A Geometric Analysis. Int J Radiat Oncol Biol Phys 2018;102:858-66.

42. De Ruysscher D, Sterpin E, Haustermans K, et al. Tumour movement in proton therapy: Solutions and remaining questions: A review. Cancers (Basel) 2015;7:1143-53.

43. Ribeiro CO, Meijers A, Korevaar EW, et al. Comprehensive 4D robustness evaluation for pencil beam scanned proton plans. Radiother Oncol 2019;136:185-9.

44. Souris K, Barragan Montero A, Janssens G, et al. Technical Note: Monte Carlo methods to comprehensively evaluate the robustness of 4D treatments in proton therapy. Med Phys 2019;46:4676-84.

45. Chargari C, Magne N, Guy JB, et al. Optimize and refine therapeutic index in radiation therapy: Overview of a century. Cancer Treat Rev 2016;45:58-67.

46. Chargari C, Levy A, Paoletti X, et al. Methodological Development of Combination Drug and Radiotherapy in Basic and Clinical Research. Clin Cancer Res 2020;26:4723-36.
Cite this article as: Botticella A, Levy A, Auzac G, Chabert I, Berthold C, Le Pechoux C. Tumour motion management in lung cancer: a narrative review. Transl Lung Cancer Res 2021;10(4):2011-2017. doi: 10.21037/tlcr-20-856 